\title{
Impact of Treatment Parameter on Blood Flow in an Atherosclerotic Artery
}

\author{
Kubugha Wilcox Bunonyo ${ }^{1, ~ *, ~ E m e k a ~ A m o s ~}{ }^{2}$ \\ ${ }^{1}$ Department of Mathematics \& Statistic, Federal University Otuoke, Yenagoa, Nigeria \\ ${ }^{2}$ Department of Mathematics, Rivers State University, Port Harcourt, Nigeria \\ Email address: \\ wilcoxbk@fuotuoke.edu.ng (K. W. Bunonyo),Amos.emeka@ust.edu.ng (E. Amos) \\ ${ }^{*}$ Corresponding author
}

\section{To cite this article:}

Kubugha Wilcox Bunonyo, Emeka Amos. Impact of Treatment Parameter on Blood Flow in an Atherosclerotic Artery. American Journal of Theoretical and Applied Statistics. Vol. 9, No. 3, 2020, pp. 74-79. doi: 10.11648/j.ajtas.20200903.17

Received: April 19, 2020; Accepted: May 7,2020; Published: May 27, 2020

\begin{abstract}
This research was carried out to investigate the impact of treatment parameter on blood flow in an atherosclerotic artery by formulating a momentum equation governing the flow in dimensional form which was scaled to dimensionless form using some important scaling parameters. The equation was solved analytical and obtained velocity profile, thereafter the volumetric flow rate, shear stress were calculated analytically and some pertinent physical parameters were obtained, finally Mathematica codes were developed to simulation the analytical results by varying the pertinent parameters to investigate the influence of the physical parameters on the blood flow profile, volumetric flow rate and the shear stress. In conclusion, it is seen that some of the pertinent parameters $R_{T}, \operatorname{Re}, D a, M, \omega, \delta$ caused the flow to improve while the others did not, taken $t=5$ seconds. This research is very helpful in providing an insight of the treatment excessive intake of fatty substance.
\end{abstract}

Keywords: Treatment, Blood, Atherosclerosis, Flow, Darcy Number, Magnetic Field

\section{Introduction}

Atherosclerosis is leading silent killer of the $21^{\text {st }}$ century, it is the constriction of and hardening of the arterial channel due to the deposition of fatty or waxy substances in the lumen. If fatty substance stream through in the lumen, there's high tendency of those substance to clog to the walls of the artery because of the pulsating nature of the heart. The heart pumps blood through the circulatory system to supply all organs and tissues of the body. If blood supply is inhibited there is bound to be pains around because of insufficient supply to the region, and once that happens around the carotid artery, there is a tendency of ischemia, many other risk factors such as hypertension and coronary artery impairment would lead to death of heart tissues, Bunonyo et al [1].

Blood is a viscous substance, it is made of the formed elements and the blood plasma, the plasma is a yellowish like fluid made up of about 55 percent of the blood volume and if the protein in the plasma is extracted it becomes serum fluid. The percentage of the red blood cells in the total blood volume is called the haematocrit. The formed elements are constitutes about 45 percent of the total blood volume, it is made of erythrocytes also called the red blood cells, the leucocytes also known as white blood cells, we also referred to the white blood cells as the defenders of human immune system to fight against viruses and other invaders, and finally we have the platelets which are also known as thrombocytes, they are helpful in the healing process of injuries or wounds. The red blood cells help in transporting hemoglobin from the lung to organs and tissues of the body for living as the key function. However, the importance of the other contributing factors can't be underemphasized, the dietary contribution to atherosclerosis is creating serious concern, the excessive fatty and cholesterol laden foods intake is alarming in this century and is a major contributing factor to atherosclerosis which is a silent killer. Nowadays, people tend to take fatty food and it is the mainstay food in their menu. Over the years, a lot of scientists and mathematicians alike have carried several researches on blood flow and atherosclerosis to investigate some cases concerning the flow of blood in human circulatory system and found some important results, and here are few of those literatures to support this research work. Ellahi [2] investigated the effect of MHD and temperature dependent viscosity of non-Newtonian nanofluid in a pipe. Bhatti et al [3] used clot model to analyze heat transfer on 
peristaltically induced movement of the particle-fluid encapsulate with resistance. The lubricant layer between red blood cells and tube walls research was carried out by Madhu et al.[4], the influence of blood flow in a large vessel on temperature distribution in hyperthermia has been developed by Lagendijk [5], Wang [6] carried out a research on blood flow in a small tube using two-fluid model. According to Ramamurthy and Shanker [7] researched on the effect of MHD on blood flow through a porous channel, they assumed blood to be a Newtonian and conducting fluid. Arterial MHD pulsatile flow of blood under periodic body acceleration was investigated by Das and Saha [8], severe stenosis causes blood supply which results inserious effect called carotid arterial blockage according to Srivastava [9], Zamir and Roach [10] examined blood flow downstream of a 2D bifurcation with a symmetrical steady flow. In $[11,12]$, Bunonyo et al. investigated the flow blood in an indented artery with heat in the presence of magnetic field. They also investigated an unsteady oscillatory coquette flow between two parallel plates with radiative heat flux. In addition, Bunonyo and Amos [13] investigated the treatment and radiation effect on blood flow stenosed artery by solving the formulated energy equation.

Kumar et al. [14], Biswas and Chakraborty [15], Chaturani and Ponalagusamy [16]) elucidated on the theoretical model of blood flow uniform artery in presence of slip velocity to study the flow parameters of blood.

After going through the aforementioned literatures, we considered the impact of treatment of blood flow in this investigation, because excessive intake of high fatty diet would lead to overweight, and overweight as results of cholesterol deposition is a risk fact for atherosclerosis which gives rise to this research using mathematical model, early detection of mild atherosclerosis and treatment is the best way to avoid serious medical condition.

\section{Mathematical Formulation}

Let's consider blood to be a Newtonian, incompressible and as a viscous fluid, flowing through an atherosclerotic artery which we assumed to be acylindrical polar channel $w^{*}\left(r^{*}, x^{*}\right)$ and is porous, where $r^{*}$ and $x^{*}$ are the direction of the flow, in addition, the flow is caused by contraction of the heart and the pressure gradient is in horizontal direction and the magnetic is applied perpendicularly to the direction of blood flow. The flow through the directional is zero and likewise the electric field, the flow is not subjected to any applied voltage. Following Bunonyo et al [1] we present the equation governing the blood flow as:

$$
\rho \frac{\partial w^{*}}{\partial t^{*}}=-\frac{\partial p^{*}}{\partial x^{*}}+\frac{\mu}{r^{*}} \frac{\partial}{\partial r^{*}}\left(r^{*} \frac{\partial w^{*}}{\partial r^{*}}\right)-\frac{\mu}{k^{*}} w^{*}-\sigma B_{0}^{2} w^{*}
$$

Subject to the boundary condition:

$$
\left.\begin{array}{lll}
\frac{\partial w^{*}}{\partial r^{*}}=0 & \text { at } & r^{*}=0 \\
w^{*}=0 & \text { at } & r^{*}=R
\end{array}\right\}
$$

The region of atherosclerosis is assumed to be:

$$
r^{*}=R_{0}-\frac{\delta^{*}}{2}\left(1+\cos 2 \frac{\pi x^{*}}{\lambda}\right)(3)
$$

where

$$
x^{*}=d_{0}+\frac{L_{0}}{2}(4)
$$

We introduce the following non-dimensional conditions in order solve equation (1) subject to the boundary condition in equation (2):

$$
\left.\begin{array}{l}
w=\frac{w^{*}}{U_{0}} ; t=\frac{t^{*} U_{0}}{R_{0}} ; D a=\frac{k^{*}}{R_{0}^{2}} ; \operatorname{Re}=\frac{U_{0} R_{0}}{v} ; M=\frac{\sigma B_{0}^{2} R_{0}^{2}}{\mu} ; \\
\mu^{*}=\frac{\mu}{\mu_{0}} ; x=\frac{x^{*}}{\lambda} ; \delta^{*}=\frac{2 \delta}{R_{T}} ; p=\frac{R_{0}^{2} p^{*}}{\rho v \lambda w_{0}} ; r=\frac{r^{*}}{R_{0}}
\end{array}\right\}
$$

Equations (1) \& (3) are transformed using equation (5), we obtain:

$$
\begin{gathered}
R e \frac{\partial w}{\partial t}=-\frac{\partial P}{\partial x}+\frac{1}{r} \frac{\partial}{\partial r}\left(r \frac{\partial w}{\partial r}\right)-\frac{1}{D a} w-M^{2} w \\
r=1-\frac{\delta}{R_{T}}(1+\cos 2 \pi x)
\end{gathered}
$$

where:

$$
x=\frac{1}{\lambda}\left(d_{0}+\frac{L_{0}}{2}\right)
$$

Subject to the boundary condition:

$$
\left.\begin{array}{lll}
\frac{\partial w}{\partial r}=0 & \text { at } & r=0 \\
w=0 & \text { at } & r=h
\end{array}\right\}
$$

\section{Method of Solution}

In order to solve the dimensionless governing equation in equation (6) subject to the boundary conditions in equation (9), as the heart pumps blood through the circulatory channel, we assume the solution to be in the form:

$$
\left.\begin{array}{l}
w=w_{0} e^{i \omega t} \\
\frac{\partial P}{\partial x}=-P_{0} e^{i \omega t}
\end{array}\right\}
$$

Substitute equation (10) into the dimensionless equation (6) and equation (9), we obtain: 


$$
\frac{1}{r} \frac{\partial}{\partial r}\left(r \frac{\partial w_{0}}{\partial r}\right)-\left(\frac{1}{D a}+M^{2}+\operatorname{Re} i \omega\right) w_{0}=0
$$

Subject to the boundary condition:

$$
\left.\begin{array}{lll}
w_{0}=0 & \text { at } & r=0 \\
w_{0}=0 & \text { at } & r=h
\end{array}\right\}
$$

Applying transformation $\xi=\frac{r}{h}$ into equation (11) and equation (12) are reduced to the following:

$$
\frac{1}{\xi} \frac{\partial}{\partial \xi}\left(\xi \frac{\partial w_{0}}{\partial \xi}\right)-h^{2}\left(\frac{1}{D a}+M^{2}+\operatorname{Re} i \omega\right) w_{0}=P_{0}
$$

Let

$$
\beta_{1}=h^{2}\left(\frac{1}{D a}+M^{2}+\operatorname{Re} i \omega\right)
$$

then equation (13) is reduced to:

$$
\left.\begin{array}{c}
\xi^{2} \frac{\partial w_{0}}{\partial \xi}+\xi \frac{\partial w_{0}}{\partial \xi}-\beta_{1} \xi^{2} w_{0}=-P_{0} \\
w_{0}=0 \quad \text { at } \quad \xi=0 \\
w_{0}=0 \quad \text { at } \quad \xi=1
\end{array}\right\}
$$

Solving the Bessel equation (14), we obtain the following:

$$
w_{0}(\xi)=A I_{0}\left(\sqrt{\beta_{1}} \xi\right)+B K_{0}\left(\sqrt{\beta_{1}} \xi\right)+\frac{P_{0}}{\beta_{1}}
$$

where $I_{0}$ and $K_{0}$ are modified Bessel function of the first kind and second kind respectively.

$B=0$ as otherwise at $r=0$, then $w_{0}(\xi)$ is not finite. So, equation (16) is reduced to:

$$
w_{0}(\xi)=A I_{0}\left(\sqrt{\beta_{1}} \xi\right)+\frac{P_{0}}{\beta_{1}}
$$

Solving equation (17) subject to equation (15) we obtain the following:

$$
w_{0}(\xi)=\frac{P_{0}}{\beta_{1}}\left(1-\frac{I_{0}\left(\sqrt{\beta_{1}} \xi\right)}{I_{0}\left(\sqrt{\beta_{1}}\right)}\right)
$$

Substituting equation (18) into equation (10), we have the following:

$$
w(\xi)=\left(\frac{P_{0}}{\beta_{1}}\left(1-\frac{I_{0}\left(\sqrt{\beta_{1}} \xi\right)}{I_{0}\left(\sqrt{\beta_{1}}\right)}\right)\right) e^{i \omega t}
$$

The shear stress can be mathematically stated as:

$$
C f=\left.\frac{\partial w(\xi)}{\partial \xi}\right|_{\xi=1}=e^{i \omega t} \frac{\partial}{\partial \xi}\left(\frac{P_{0}}{\beta_{1}}\left(1-\frac{I_{0}\left(\sqrt{\beta_{1}} \xi\right)}{I_{0}\left(\sqrt{\beta_{1}}\right)}\right)\right)_{\xi=1}=\frac{P_{0}}{\sqrt{\beta_{1}}}\left(\frac{I_{1}\left(\sqrt{\beta_{1}}\right)}{I_{0}\left(\sqrt{\beta_{1}}\right)}\right) e^{i \omega t}
$$

The blood flow rate through an artery is mathematically stated as:

$$
\begin{gathered}
Q=2 \pi \int_{0}^{\xi=1} \xi w(\xi) d \xi=2 \pi e^{i \omega t} \int_{0}^{\xi=1}\left(\frac{P_{0}}{\beta_{1}}\left(\xi-\frac{\xi I_{0}\left(\sqrt{\beta_{1}} \xi\right)}{I_{0}\left(\sqrt{\beta_{1}}\right)}\right)\right) d \xi \\
Q=2 \pi e^{i \omega t}\left(\frac{P_{0}}{\beta_{1}}\left(\frac{1}{2}-\frac{I_{1}\left(\sqrt{\beta_{1}}\right)}{\beta_{1} I_{0}\left(\sqrt{\beta_{1}}\right)}\right)\right)
\end{gathered}
$$

\section{Results}

The numerical simulation was done with Mathematica codes developed by the authors for analytical results in equations (19), (20) and (22) with the pertinent parameter values used are $R_{T}=1, \delta=2, M=0.3, D a=0.4, \omega=5, x=0.6, R e=2$ for $t=5$ seconds. In order to see the effect of the parameters values, they are varied within a specified range as seen with graphical results presented below:

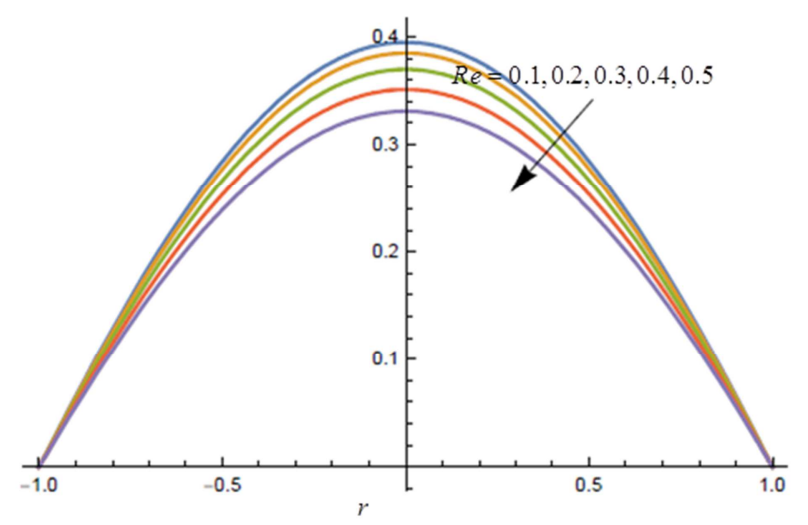

Figure 1. The effect of the increase in Re on blood flow, where other parameters values are: $R_{T}=0.2, \delta=2, M=0.3, D a=0.4, \omega=5, x=0.6$.

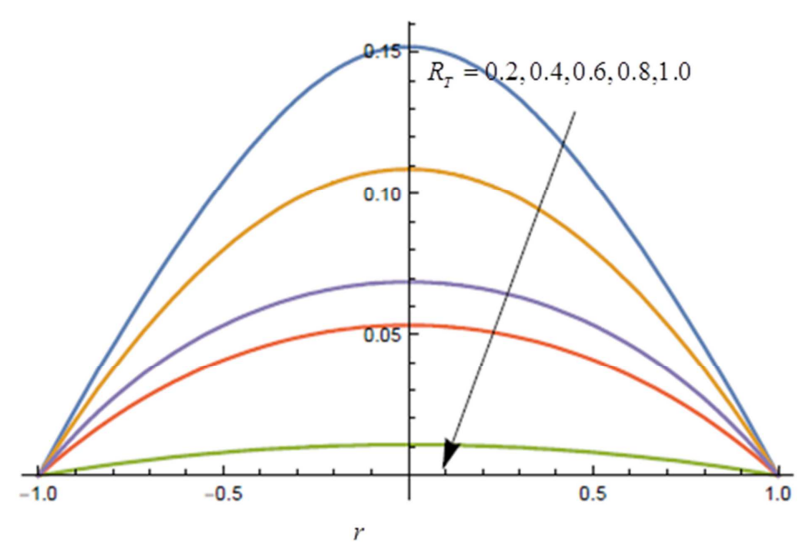

Figure 2. The effect of the increase in $R_{T}$ on blood flow, where other parameters values are: $\operatorname{Re}=2, \delta=2, M=0.3, D a=0.4, \omega=5, x=0.6$. 


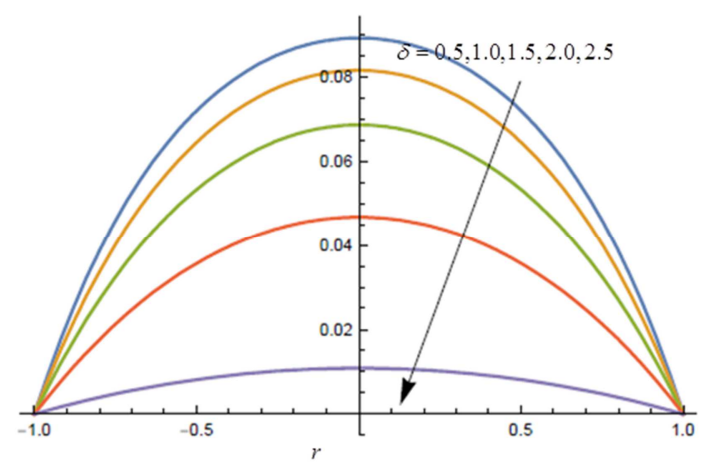

Figure 3. The effect of the increase in $\delta$ on blood flow, where other parameters values are: $R_{T}=0.5, R e=2, M=0.3, D a=0.4, \omega=5, x=0.6$.

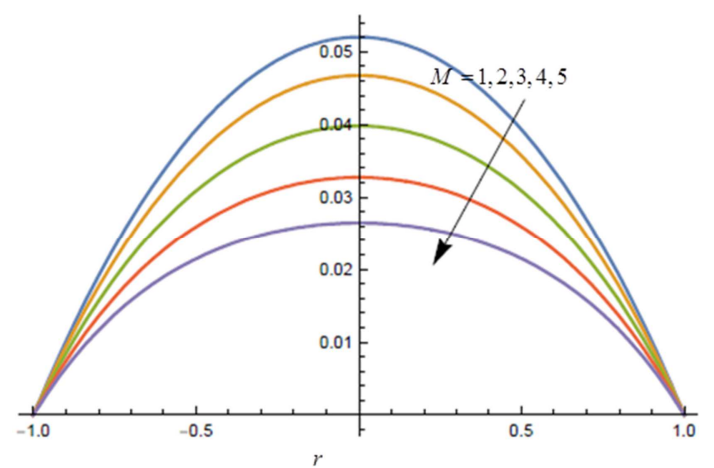

Figure 4. The effect of the increase in Re on blood flow, where other parameters values are: $R_{T}=0.5, \delta=2, R e=2, D a=0.4, \omega=5, x=0.6$.

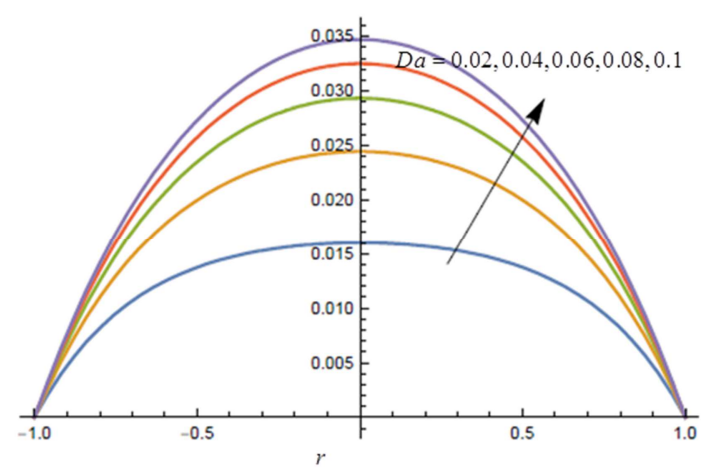

Figure 5. The effect of the increase in Da on blood flow, where other parameters values are: $R_{T}=0.5, \delta=2, M=2, R e=0.5, \omega=5, x=0.6$.

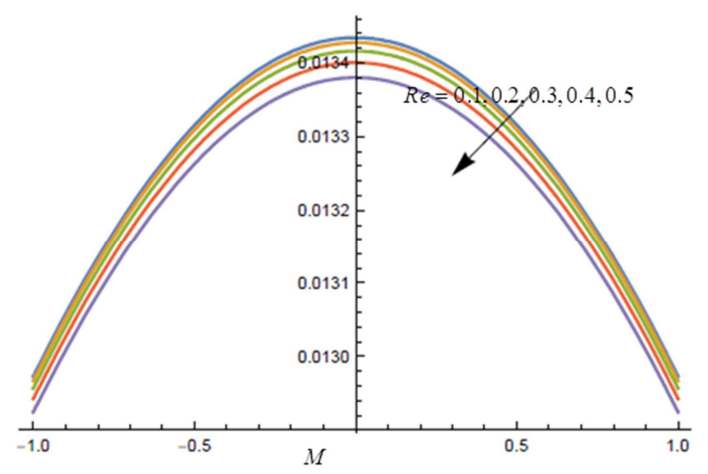

Figure 6. The effect of the increase in Re on blood flow, where other parameters values are: $R_{T}=0.5, \delta=2, R e=2, D a=0.4, \omega=5, x=0.6$.

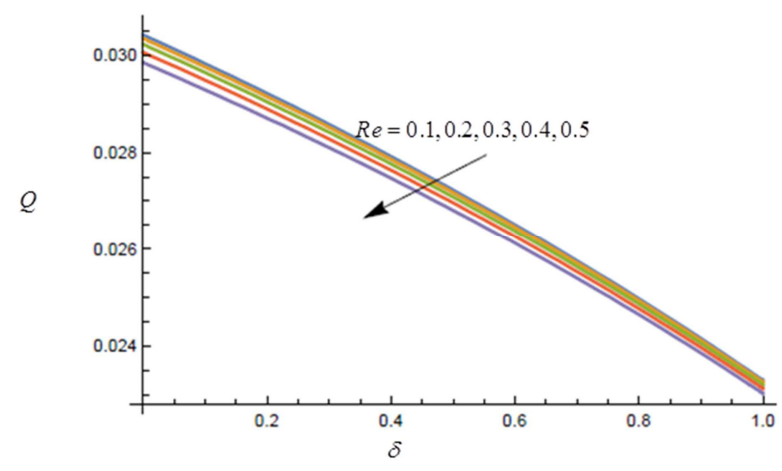

Figure 7. The effect of the increase in Re on blood flow, where other parameters values are: $R_{T}=0.5, \delta=2, R e=2, D a=0.4, \omega=5, x=0.6$.

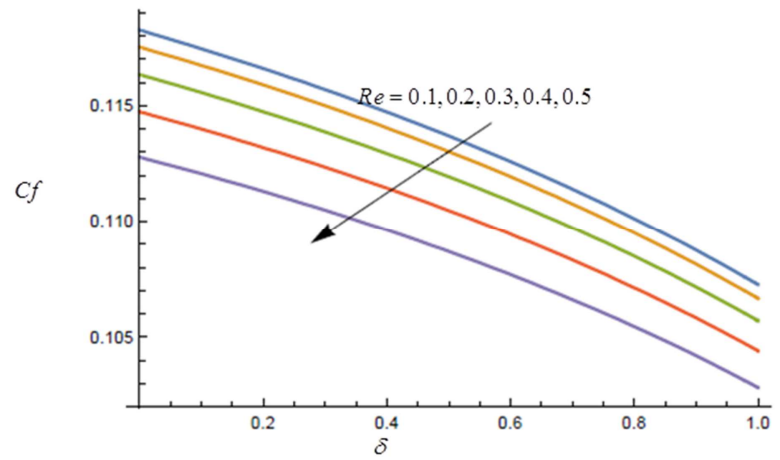

Figure 8. The shear stress while Re increases, where other parameters values are: $R_{T}=0.5, \delta=2, R e=2, D a=0.4, \omega=5, x=0.6$.

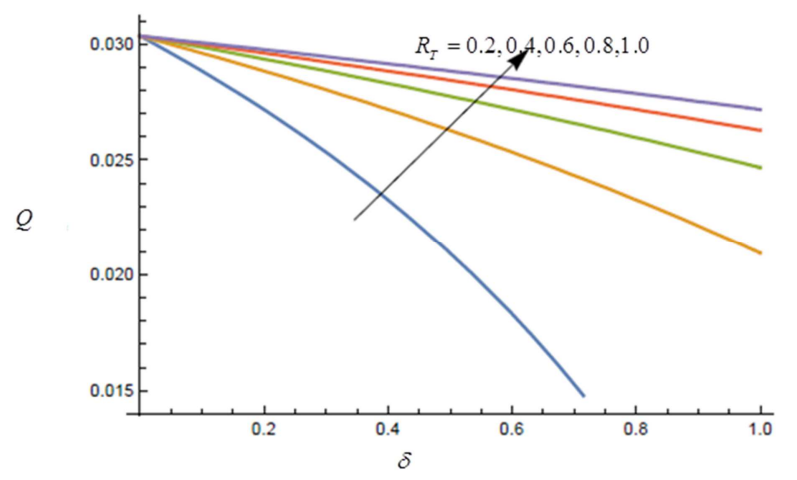

Figure 9. The effect of the increase in $R_{T}$ on blood flow, where other parameters values are: $R_{T}=0.5, \delta=2, R e=2, D a=0.4, \omega=5, x=0.6$.

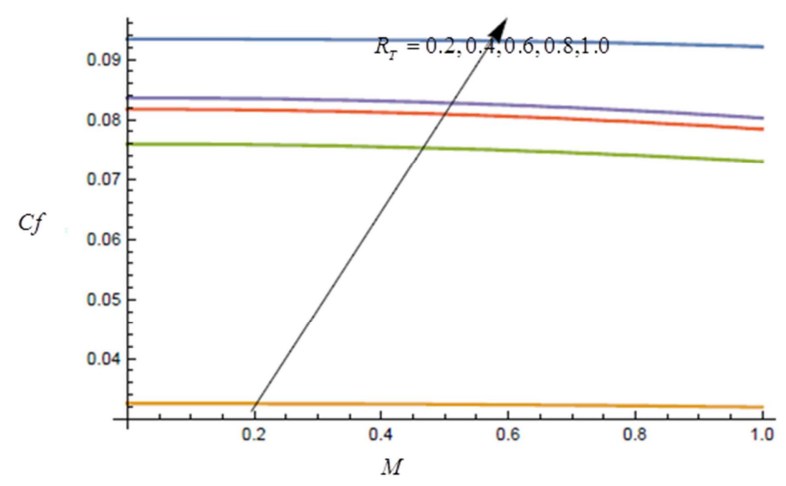

Figure 10. The shear stress while $R_{T}$ increases, where other parameters values are: $R_{T}=0.5, \delta=2, R e=2, D a=0.4, \omega=5, x=0.6$. 


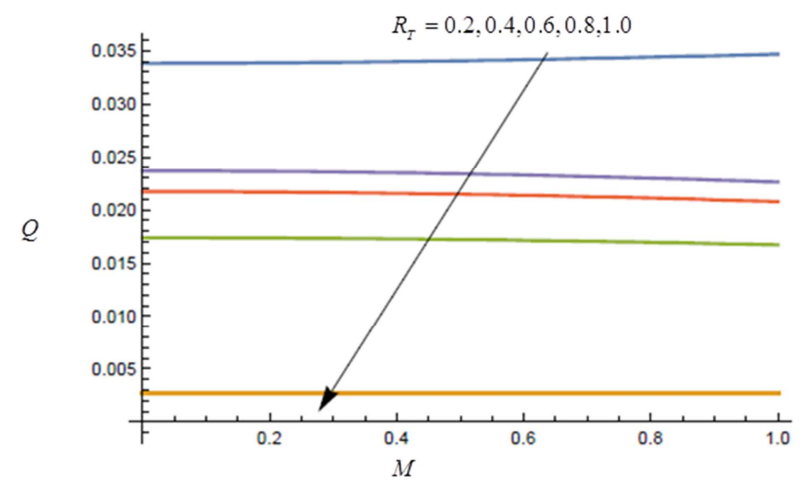

Figure 11. The effect of the increase in $R_{T}$ on blood flow rate, where other parameters values are: $R_{T}=2, \delta=2, D a=0.4, \omega=5, x=0.6$.

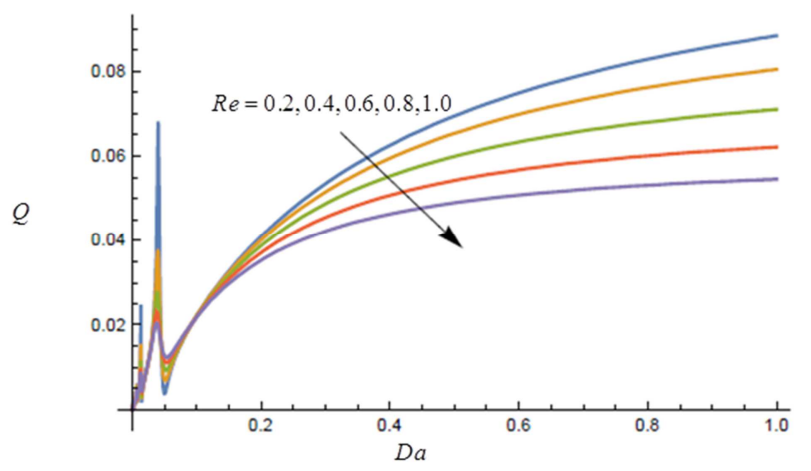

Figure 12. The effect of the increase in Re on blood flow, where other parameters values are: $R_{T}=0.2, \delta=2, M=3, \omega=5, x=0.6$.

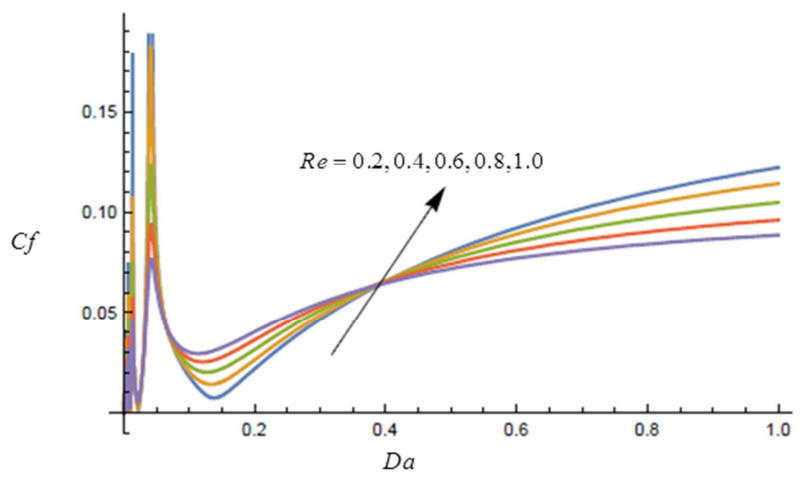

Figure 13. The shear stress while Re increases, where other parameters values are: $R_{T}=0.2, \delta=2, M=3, \omega=5, x=0.6$.

\section{Discussion}

In this section, we shall discuss the results obtained in the preceding section after carrying out a thorough numerical simulation.

It is clearly seen in Figure 1 the influence of $R e$ on blood velocity, we noticed that as the parameter values increases, the velocity profile decreases as depicted by the different maximum values. In addition, it is seen that the velocity is maximum at the centre with different centre maximums in view of the Reynolds's number values. But as the stenosed region or the wall thickness gets larger, the velocity decreases and arrived at zero when $r=1$, which is technically in conformity with existing laws of physics.

Following the numerically simulated result in the preceding section, Figure 2 shows the influence of the treatment parameter $R_{T}$ on the blood velocity in the arterial channel. It is noticed that the treatment parameter increase resulted to blood velocity decreases. The treatment is intended to control the cholesterol build up in the lumen of the arterial segment. The obtained result is in agreement with physical laws because the velocity is maximum according to the dosage of the treatment parameter values at the centre when $r=0$, and after several minutes resulted to zero at $r=1$.

The growth of the height of stenosis causes a lot of cardiovascular challenges as it is seen it Figure 3. The figure illustrates that as the height of stenosis increases the blood flow is retarded, and that retardation could cause serious circulatory challenges because it is a clear indication that the tissues and organs are starved of oxygen rich blood for human well-being. This result is in conformity with existing laws based on fact that the velocity maximum at the centre and minimum elsewhere.

The applied magnetic field is very important is blood flow analysis and detection of tumor such as using MRI and ECG. It is noticed in Figure 4 that as the magnetic field intensity increases, the blood velocity is retarded. This is so because when magnetic field is applied to a moving electrically conducting fluid such as blood, it generates a force called Lorentz force which retards/opposes the flow of blood.

Porosity of a substance helps in letting in more of it as the case in Figure 5. The figure illustrates that an increase in the pores of the region, that is, the Darcy parameter leads to an increase in blood velocity. This is seen by the increasing maximum heights of the blood velocity at the centre and this result is also in conformity with set boundary conditions and some natural laws.

The influence of pertinent parameters on flow rate $Q$ and shear stress $C f$ can be seen in Figure 6 - Figure 13. The parameters influence the flow rate and stress coefficient by increasing and decreasing it at different level.

\section{Conclusion}

Having formulated a mathematical model to investigate the impact of the treatment parameter on blood flow in an atherosclerotic artery successfully, solved the model analytically, carried out numerical simulation using scientific software called Mathematica and results well discussed based on their merits, here we can conclude on some of our findings:

The Reynolds's number influences the blood flow to decrease while other valuable parameters are held.

The treatment parameter causes retardation of blood flow profile while other parameters are held. This is because the influence of the magnetic field parameters could not be undermined.

The height of stenosis is major channel is well-being of an individual because it cause a reduction in blood flow, thereby starving the hemoglobin needs of the tissues and organs for survival. 
The magnetic field intensity retards blood flow in the system and that can be helpful under carefully monitored study to detect tumor growth or to know the level of stenosis in the lumen of an arterial segment.

The porosity of the channel is very helpful in the sense that the flow is improved when the porosity is increased. So, there is need to maintain some level of porosity that was achieved with the treatment parameter involvement.

We can also conclude that the pertinent parameters greatly influenced the flow rate and the shear stress coefficient as seen.

\section{Nomenclature}

$r^{*}$ : Dimensional perpendicular distance

$r$ : Dimensionless perpendicular distance

$x^{*}$ : Dimensional horizontal distance

$x$ : Dimensionless horizontal distance

$R_{0}$ : Radius of normal arterial segment

$R$ : Radius of abnormal arterial segment

$R e$ : Reynolds number

$\lambda, L_{0}$ : Length of arterial segment

$w^{*}$ : Dimensional blood velocity profile

$w$ : Dimensionless blood velocity profile

$\delta^{*}$ : Maximum height of stenosis

$R_{T}$ : Treatment parameter

$h$ : Boundary thickness

$d_{0}$ : Onset of stenosis

$t$ : Time parameter

$\mu$ : Blood dynamic viscosity

$\rho:$ Blood density

$\omega$ : Oscillatory frequency parameter

\section{Conflict of Interests}

The authors declare that there is no conflict of interests regarding the publication of this paper.

\section{Acknowledgements}

The authors would like to thank the reviewers and editor for their valuable comments in improving this paper.

\section{References}

[1] Bunonyo, K. W., Israel-Cookey, C., \& Amos, E. (2018). Modeling of Blood Flow through Stenosed Artery with Heat in the Presence of Magnetic Field. Asian Research Journal of Mathematics, 1-14.

[2] Ellahi, R. (2013). The effects of MHD and temperature dependent viscosity on the flow of non-Newtonian nanofluid in a pipe: analytical solutions. Applied Mathematical Modelling, 37 (3), 1451-1467.

[3] Bhatti, M. M., Zeeshan, A., \&Ellahi, R. (2016). Heat transfer analysis on peristaltically induced motion of particle-fluid suspension with variable viscosity: clot blood model. Computer Methods and Programs in Biomedicine, 137, 115124.

[4] Sharma, G. C., Jain, M., \& Singh, A. (2009). Mathematical Analysis of MHD Flow of Blood in Very Narrow Capillaries. International Journal of Engineering, 22 (3), 307-315.

[5] Lagendijk, J. J. W. (1982). The influence of bloodflow in large vessels on the temperature distribution in hyperthermia. Physics in Medicine \& Biology, 27 (1), 17.

[6] Wang, Q. Q., Ping, B. H., Xu, Q. B., \& Wang, W. (2008). Rheological effects of blood in a nonplanar distal end-to-side anastomosis. Journal of biomechanical engineering, 130 (5).

[7] Ramamurthy, G., \& Shanker, B. (1994). Magnetohydrodynamic effects on blood flow through a porous channel. Medical and Biological Engineering and Computing, 32 (6), 655-659.

[8] Das, K., \&Saha, G. C. (2009). Arterial MHD pulsatile flow of blood under periodic body acceleration. Bulletin of Society of Mathematicians Banja Luka, 16, 21-42.

[9] Srivastava, N. (2014). Analysis of flow characteristics of the blood flowing through an inclined tapered porous artery with mild stenosis under the influence of an inclined magnetic field. Journal of Biophysics, 2014.

[10] Zamir, M., \& Roach, M. R. (1973). Blood flow downstream of a two-dimensional bifurcation. Journal of theoretical biology, $42(1), 33-48$

[11] Bunonyo, K. W., Amos, E., \& Eli, I. C. (2018). Unsteady oscillatory couette flow between vertical parallel plates with constant radiative heat flux. Asian Research Journal of Mathematics, 1-11.

[12] Bunonyo, K. W., Israel-Cookey, C., \& Amos, E. (2017). MHD Oscillatory Flow of Jeffrey Fluid in an Indented Artery with Heat Source. Asian Research Journal of Mathematics, 1-13.

[13] Bunonyo, K. W., \& Amos, E. (2020). "Investigation of the Treatment and Radiation Effects on Oscillatory Blood Flow through a Stenosed Artery." American Journal of Engineering Research (AJER), vol. 9 (04), pp. 253-259.

[14] Kumar, S., \& Diwakar, C. (2013). A mathematical model of power law fluid with an application of blood flow through an artery with stenosis. Advances in Applied Mathematically BioSciences, 4 (2), 51-61.

[15] Biswas, D., \& Chakraborty, U. S. (2010). Two-layered pulsatile blood flow in a stenosed artery with body acceleration and slip at wall. Applications and Applied Mathematics: An International Journal (AAM), 5 (10), 1400-1417.

[16] Chaturani, P., \& Ponnalagar Samy, R. (1985). A study of nonNewtonian aspects of blood flow through stenosed arteries and its applications in arterial diseases. Biorheology, 22 (6), 521-531. 